\title{
SHOCK ACCELERATION OF SOLAR ENERGETIC PROTONS: THE FIRST 10 MINUTES
}

\author{
C. K. $\mathrm{Ng}^{1}$ and D. V. Reames \\ NASA Goddard Space Flight Center, Greenbelt, MD 20771 \\ Chee.Ng@nasa.gov, Donald.V.Reames@nasa.gov
}

\begin{abstract}
Proton acceleration at a parallel coronal shock is modeled with self-consistent Alfvén wave excitation and shock transmission. 18 - $50 \mathrm{keV}$ seed protons at $0.1 \%$ of plasma proton density are accelerated in 10 minutes to a power-law intensity spectrum rolling over at $300 \mathrm{MeV}$ by a $2500 \mathrm{~km} \mathrm{~s}^{-1}$ shock traveling outward from $3.5 r_{\odot}$, for typical coronal conditions and low ambient wave intensities. Interaction of high-energy protons of large pitch-angles with Alfvén waves amplified by low-energy protons of small pitch angles is key to rapid acceleration. Shock acceleration is not significantly retarded by sunward streaming protons interacting with downstream waves. There is no significant second-order Fermi acceleration.
\end{abstract}

Subject headings: acceleration of particles — shock waves — Sun: coronal mass ejections (CMEs)

\section{INTRODUCTION}

The acceleration of solar energetic particles (SEPs) by coronal mass ejection (CME) driven shocks is an outstanding problem in space weather and astrophysics. Observations indicate that SEPs are accelerated to $\mathrm{GeVs}$ in $\lesssim 10$ minutes after CME launch in many events. This short time scale requires mean free paths $\lambda \lesssim 1 \times 10^{-6}$ AU in diffusive shock acceleration models, in apparent contradiction with $\lambda \gtrsim 0.2$ AU deduced from interplanetary (IP) transport of SEPs (e.g., Bieber et al. 1994). Waves excited by streaming SEPs resolve this paradox. Self-amplified Alfvén waves were invoked in the confinement (Wentzel 1974) and diffusive shock acceleration of cosmic rays (Bell 1978). Steady-state models with self-consistent Alfvén waves were applied successfully at the Earth's bow shock and traveling

\footnotetext{
${ }^{1}$ Department of Astronomy, University of Maryland, College Park, MD20742
} 
IP shocks (Lee 1982, 1983, 2005; Gordon et al. 1999). Ng \& Reames (1994) showed theoretically that self-amplified waves limit SEP intensity at 1 AU in large events. Reames \& Ng (1998) inferred streaming-limited SEP intensities and their radial dependence from Helios and GOES data. Ng et al. (1999) and Reames et al. (2000) showed how proton-amplified waves control abundance variation in SEP events. Can streaming SEPs amplify Alfvén waves upstream of CME-driven coronal shocks by a factor $\gtrsim 10^{5}$ in a few minutes and to what energy are the SEPs accelerated? The answer requires a time-dependent model that treats self-consistently the nonlinear, coupled evolution of SEPs and Alfvén waves.

SEP acceleration at a CME-driven shock is a very complex phenomenon (Lee 2005; Cliver et al. 2004; Reames 1999). Researchers have studied different aspects theoretically with various simplifying assumptions, using various analytical and numerical techniques. Significant progress has been made with many issues under lively debate. The reader is referred to Tylka \& Lee (2006), Zank et al. (2006), and Giacalone (2005) for the effects of shock geometry; Zank et al. (2007) for the excellent work performed by their group; and Giacalone \& Neugebauer (2008) for the effects of a rippled shock.

In this Letter we address the question of how rapid and to what energy a fast parallel shock accelerates SEPs in typical coronal conditions by solving numerically the coupled evolution of SEPs and Alfvén waves. We also address the issues of whether shock acceleration is significantly retarded by SEP damping of outward waves and excitation of inward waves downstream of the shock (Vainio 2001; Ng 2007) and whether second-order Fermi acceleration plays a significant role.

Our model includes in SEP transport: focusing, convection, adiabatic deceleration, pitch-angle scattering, and momentum diffusion due to scattering by counter-streaming Alfvén waves. Treatment of Alfvén waves includes propagation, SEP-driven growth and damping, and shock transmission (Vainio \& Schlickeiser 1999). A unique feature of the numerical model is the implementation of the pitch-angle dependent cyclotron resonance condition to calculate self-consistent SEP scattering and Alfvén wave growth. This feature is essential for understanding how acceleration is initiated for SEPs above the "knee" energy $E_{k n e e}$. Momentum diffusion governed by the diffusion tensor elements $D_{\mu \mu}, D_{\mu P}=D_{P \mu}$, $D_{P P}$ (Ng et al. 2003) is solved by using an iterative method more accurate than the direct method employed in $\mathrm{Ng}(2007)$. The model now covers larger rigidity $(P)$ and wavenumber $(k)$ ranges to reach higher energy.

Our model accounts for wave evolution time and does not adopt an instantaneous steadystate upstream (US) wave spectrum (Li et al. 2005). The model of Vainio \& Laitinen (2007) treats US wave growth and accelerates protons to tens of $\mathrm{MeV}$ in 10 minutes. They exclude the downstream (DS) region by reflecting SEPs from the shock statistically. The DS region 
is an important part of our consideration.

We report results for a strong $2500 \mathrm{~km} / \mathrm{s}$ shock starting at $3.5 r_{\odot}$. Cliver et al. (2004) have reported that shock acceleration is most efficient above $\sim 3 r_{\odot}$. Studies on the dependence on shock, plasma, and turbulence parameters will be published in a full paper. We describe the model in Section 2, present the results in Section 3, and close with a discussion in Section 4 .

\section{THE MODEL}

The time-dependent nonlinear model of $\mathrm{Ng}$ et al. (2003) is generalized to treat SEP shock acceleration and shock transmission of Alfvén waves in a computational box comoving with the shock. Radial geometry is assumed with mean magnetic field $B=B_{0}\left(r_{0} / r\right)^{2}$, plasma proton density $n_{\mathrm{H}}=n_{\mathrm{H}, 0}\left(r_{0} / r\right)^{2}$, hence Alfvén velocity $V_{\mathrm{A}}=V_{\mathrm{A}, 0}\left(r_{0} / r\right)$, where $B_{0}$, $n_{\mathrm{H}, 0}$, and $V_{\mathrm{A}, 0}=B_{0} / \sqrt{4 \pi n_{\mathrm{H}, 0}}$ are values at the reference radius $r_{0}=3.5 r_{\odot}$. The evolution of the energetic proton distribution is governed by:

$$
\begin{gathered}
\partial_{t} F+\partial_{x}(\dot{x} F)+\partial_{\mu}(\dot{\mu} F)+\partial_{\varrho}(\dot{\varrho} F)=\left[\begin{array}{c}
\partial_{\mu} \\
\partial_{\varrho}
\end{array}\right]^{T}\left[\begin{array}{c}
D_{\mu \mu} D_{\mu \varrho} \\
D_{\varrho \mu} D_{\varrho \varrho}
\end{array}\right]\left[\begin{array}{c}
\partial_{\mu}(\chi F) \\
P^{3} \partial_{\varrho}\left(\chi F P^{-3}\right)
\end{array}\right], \\
\dot{x}=\chi\left(\mu v+W_{+}\right)-V_{\mathrm{sh}}, \\
\dot{\mu}=\left(1-\mu^{2}\right)\left[r^{-1} \chi\left(v+\mu W_{+}\right)-v^{-1} d W_{+} / d t\right] \\
\dot{\varrho}=\dot{P} / P=-r^{-1} \chi W_{+}\left(1-\mu^{2}\right)-\mu v^{-1} d W_{+} / d t
\end{gathered}
$$

where $F(P, \mu, x, t)=\left(B_{0} / B\right)\left(P / P_{0}\right)^{3} f(P, \mu, x, t) ; f=$ proton phase-space density in mixed coordinates: distance $x$ from shock and time $t$ in the Sun's inertial frame; $P$, proton velocity $v$, and pitch-angle cosine $\mu$ in the local outward wave frame moving with velocity $W_{+}(x, t)=$ $V_{\mathrm{sw}}+V_{\mathrm{A}}$ relative to the Sun. $d W_{+} / d t \equiv \partial_{t} W_{+}+\dot{x} \partial_{x} W_{+} ; \chi=\left(1+\mu v W_{+} / c^{2}\right)^{-1} ; \varrho=\ln \left(P / P_{0}\right) ;$ $P_{0}=$ constant; $D_{\mu \varrho}=D_{\varrho \mu}=P^{-1} D_{\mu P}, D_{\varrho \varrho}=P^{-2} D_{P P} ; r(x, t)=x+r_{\mathrm{sh}}(t) ; r_{\mathrm{sh}}(t)=\operatorname{shock}$ radius; $V_{\mathrm{sh}}=$ constant shock velocity. The plasma velocity $V_{\mathrm{sw}}(x)=V_{d}$ at $x<0$ (DS) and $V_{\mathrm{sw}}(x)=V_{u}$ at $x>0$ (US). Besides focusing and adiabatic deceleration, equations (3) and (4) include the differential frame transformation of $(\mu, P)$ following particle motion. The shock jump in $W_{+}(r, t)$ gives a $P$-increment for almost all shock-crossing particles (eq. [4]).

We consider only right- and left-hand circularly polarized, inward and outward parallel propagating Alfvén waves. The ambient outward wave magnetic intensities $I^{\mathrm{R}+}=I^{\mathrm{L}+} \propto k^{-\delta}$ are specified for US and DS separately via steady-state solutions to the wave kinetic equation (Ng et al. 2003). As outward waves dominate near the Sun, we specify $I^{\mathrm{R}-}=I^{\mathrm{L}-}=0.05 I^{\mathrm{R}+}$. $I^{\sigma}, n_{\mathrm{H}}$ and $V_{\mathrm{A}}$ are then modified for the shocked DS region assuming 10 s shock transmission 
before seed-particle injection at $t=0$. Wave evolution is governed by the wave kinetic equation

$$
\partial_{t} \Psi_{\sigma}+\partial_{x}\left[\left(W_{\sigma}-V_{\mathrm{sh}}\right) \Psi_{\sigma}\right]+\eta \partial_{\eta}\left(V_{\eta}^{\sigma} \Psi_{\sigma}\right)=\gamma_{\sigma} \Psi_{\sigma}
$$

with wave action density $\Psi_{\sigma}(\eta, x, t)=2 I^{\sigma}(\eta, x, t) \eta\left|W_{\sigma}\right| /\left(\eta_{0} V_{\mathrm{A}}\right), \eta=k / B, \eta_{0}=$ constant, $V_{\eta}^{\sigma}=2 W_{\sigma} / r-d W_{\sigma} / d r, \gamma_{\sigma}=$ fractional growth rate of $I^{\sigma}, W_{\sigma}=W_{+}$for $\sigma=R+, L+$, and $W_{\sigma}=W_{-}=V_{\mathrm{sw}}(r, t)-V_{\mathrm{A}}(r, t)$ for $\sigma=R-, L-$. Equations (1) and (5) are coupled via

$$
\begin{gathered}
D_{\mu \mu}=\sum_{\sigma} D_{\mu \mu}^{\sigma}=\sum_{\sigma} \frac{v^{2}}{4 P^{2}} \int d k R_{\mu \mu}^{\sigma} I^{\sigma}, \\
\gamma_{\sigma}(k, r, t)=2 \pi^{2} c e^{3} g_{\sigma} V_{\mathrm{A}} \iint d \mu d P R_{\mu \mu}^{\sigma} G f,
\end{gathered}
$$

where $G f \equiv P^{3}\left[\mathcal{E}\left(1-\mu V_{\sigma} / v\right)\right]^{-2}\left[\partial_{\mu} f-\left(V_{\sigma} / v\right)\left(\mu \partial_{\mu} f-\partial_{\varrho} f\right)\right] ; \mathcal{E}=$ total particle energy; $g_{\sigma}=1, V_{\sigma}=0$ for $\sigma=R+, L+$; and $g_{\sigma}=-1, V_{\sigma}=-2 V_{\mathrm{A}}$ for $\sigma=R-, L-$. The waveparticle resonance function $R_{\mu \mu}^{\sigma}\left(\mu, P, k, V_{\mathrm{A}}, B\right)$ (Ng \& Reames 1995; Ng et al. 2003) includes minimal broadening of the quasilinear resonance condition $k=B /\left[P\left(\mu-V_{\sigma} / v\right)\right]$.

At the initial shock location at $r=3.5 r_{\odot}$, we specify $V_{u}=83 \mathrm{~km} \mathrm{~s}^{-1}, n_{\mathrm{H}, 0}=2 \times 10^{5}$ $\mathrm{cm}^{-3}, V_{\mathrm{A}, 0}=700 \mathrm{~km} \mathrm{~s}^{-1}$ from the semi-empirical models of Guhathakurta et al. (1999). Hence $B_{0}=0.143$ gauss, consistent with results from gyrosynchrotron emission (Dulk \& McLean 1978). Prescribed ambient $I^{\sigma} \propto k^{-1.5}$ gives $\lambda=0.23 \mathrm{AU}$ at $1 \mathrm{MeV}(0.68 \mathrm{AU}$ at $100 \mathrm{MeV}$ ). Constant $V_{u}, V_{\mathrm{d}}=1880 \mathrm{~km} \mathrm{~s}^{-1}$, and $V_{\mathrm{sh}}=2500 \mathrm{~km} \mathrm{~s}^{-1}$ in the Sun's inertial frame are assumed, giving a fluid compression ratio $c_{f}=3.895$ and an initial Alfvén Mach number $M_{A}=3.45$ increasing to 5.56 at $t=10 \mathrm{~min}$, when $r_{\mathrm{sh}}=5.65 r_{\odot}$. The relation between $c_{f}, M_{A}$, plasma beta $=0.1$, and relative wave amplitude (Vainio \& Schlickeiser 1999) is satisfied at $t=0$ but not at $t>0$. The back reaction of SEPs and amplified waves on shock evolution is not considered.

For quasi-parallel shocks, Zank et al. (2001) calculate that $2 \%$ of the solar-wind protons are reflected by cross-shock electric potential to $\gtrsim 3 E_{\text {ramp }}$. Here $3 E_{\text {ramp }}=90 \mathrm{keV}$. From the observed post-shock proton distribution in the August 27, 1978 event (Gosling et al. 1981, Figs. 4 and 6), we find tail density above $18 \mathrm{keV}$ is a fraction $b=0.0002$ of the observed $n_{\mathrm{H}}=25 \mathrm{~cm}^{-3}$. Larger $b$ is expected in the strongly heated plasma DS of the $2500 \mathrm{~km} \mathrm{~s}^{-1}$ shock. There may also be suprathermal remnants from previous SEP events (Mason et al. 1999; Tylka et al. 2001). Guided by the above, we prescribe DS isotropic seed protons

$$
f_{\mathrm{inj}}=b n_{\mathrm{H}}\left(x_{0}\right)(\alpha-3) P^{-\alpha} /\left\{4 \pi\left(P_{a}^{3-\alpha}-P_{b}^{3-\alpha}\right)\right\},
$$

at $P_{a}<P<P_{b}$, with injection fraction $b=0.001, \alpha=5, P_{a}=6 \mathrm{MV}(18 \mathrm{keV})$, and $P_{b}=9.8 \mathrm{MV}(50 \mathrm{keV})$. Note that $P_{b}<P_{1}=10.4 \mathrm{MV}$, the lowest grid rigidity, so the DS seeds emerge US as outward proton beams from 10.4 to $14.7 \mathrm{MV}$ (57 to $115 \mathrm{keV}$ ). 
Each US wave $I_{u}\left(k_{u}\right)$ crossing the shock is converted to two DS waves $I_{d}\left(k_{d}\right)$ of the same helicity, taking account of shock amplification and wavenumber increase:

$$
I_{d}\left(k_{d}\right) / I_{u}\left(k_{u}\right)=\left(1+\zeta_{u} \zeta_{d} / \sqrt{c_{f}}\right)^{2} c_{w} / 4
$$

with wave compression ratio $c_{w} \equiv k_{d} / k_{u}=c_{f}\left(M_{A}-\zeta_{u}\right) /\left(M_{A}-\zeta_{d} \sqrt{c_{f}}\right), \zeta_{u}= \pm 1$ and $\zeta_{d}= \pm 1$ for outward (inward) US and DS waves (Vainio \& Schlickeiser 1999; Webb et al. 1999). Since wave growth is limited by nonlinear processes, we impose $I^{\sigma}(k) \leq I_{\text {sat }}(k)=B^{2} /(3 \pi k)$ so that $\lambda \gtrsim 3 r_{g}$. Shock transmission of US outward waves produces only a small fraction (5\%) of DS inward wave intensity. In applying the above limit, we preserve the ratio of transmitted inward and outward waves.

The numerical grid is: $x_{j}=2.15 \times 10^{-4}(j-1 / 2) r_{\odot}, j=-200: 800 ; \mu_{i}=0.05(\mathrm{i}+1 / 2)$, $i=-20: 19 ; \varrho_{\ell}=\varrho_{1}+0.08664(\ell-1), \ell=1: 60, P_{1}=10.38 \mathrm{MV} ;$ and $\log \eta_{m}=\log \eta_{1}+$ $0.05(m-1), m=1: 58, \eta_{1}=5.465 \times 10^{-4} \mathrm{MV}^{-1}$. At the boundaries, exterior $\mathrm{F}=$ interior F. At the outer boundary, exterior $I^{\sigma}=$ interior $I^{\sigma}$. The $\mu$ and $P$ increments of shockcrossing protons are evaluated by frame transformation. Equations (1) and (5) are solved with dynamic time steps using operator splitting and locally one-dimensional differencing (Ng et al. 2003); ( $\mu, P$ )-diffusion is solved iteratively.

\section{RESULTS}

In $600 \mathrm{~s}$, the $2500 \mathrm{~km} \mathrm{~s}^{-1}$ parallel shock accelerates 18 - $50 \mathrm{keV}$ DS seed protons to a power-law intensity spectrum $j_{E}$ extending to $E_{\text {knee }} \sim 300 \mathrm{MeV}$ (Fig. 1a). The energy spectral index of 0.8 is consistent with $c_{w}=6.46$ decreasing to 4.95 between US and DS outward Alfvén waves. At $12.3 \mathrm{MeV}$, the $j_{E}$ spatial profile rises swiftly from a spike at $180 \mathrm{~s}$ to the steady-state form (Lee 1983): a DS plateau and an US exponential tail at $t>250 \mathrm{~s}$ (Fig. 1b). This implies negligible second-order Fermi acceleration, consistent with no enhanced inward and outward waves in proximity.

Streaming SEPs US rapidly amplify outward waves and damp inward waves. Wave growth extends to low wavenumber $k$ (Fig. 2a) as acceleration proceeds to high energy. The amplified waves enhance scattering, providing a positive feedback that rapidly bootstraps acceleration. The DS outward waves derive from shock-transmitted US outward waves and are shifted to higher $k$ (Fig. 2b; cf. Fig. 2a). The much weaker DS inward waves (not shown) are $k$-shifted by a smaller amount. The $k$-shifted enhanced DS waves thus resonate with SEPs of lower values of $P\left(\mu-V_{\sigma} / v\right)$. The effect is seen by comparing $D_{\mu \mu}$ US and DS at $\mathrm{t}=576 \mathrm{~s}$ at five rigidities from 140 to $1449 \mathrm{MV}$ (Figs. 2c, 2d). The hump in each $D_{\mu \mu}$ curve originates from proton-amplified US waves and occupy a smaller $\mu$ range DS than US 
at $P \geq 1117 \mathrm{MV}$. At $P \leq 305 \mathrm{MV}$, however, the DS waves at $k$ resonant with large $\mu$ are amplified by US SEPs of higher $P$, so the DS humps are also wide.

How acceleration begins can be seen in the evolution of US and DS $\mu$-distributions (Figs. 2e, 2f). The scale factors chart the course. SEPs at $E>E_{\text {knee }}$ are first accelerated at the $D_{\mu \mu}$ hump at small $|\mu|$, where the resonant waves are available. Some accelerated SEPs US scatter to larger $\mu$ and excite US waves at smaller $k$, which are then transmitted DS. The result is that the DS $D_{\mu \mu}$ hump widens and acceleration extends to higher energy. The $\mu$-dependent resonance condition $k \sim B /\left[P\left(\mu-V_{\sigma} / v\right)\right]$ is crucial in this process. It allows waves excited by low $\mathrm{P}$ large $\mu \mathrm{SEPs}$ to scatter high $\mathrm{P}$ small $\mu$ SEPs (and vice versa). At $t=0, I^{\mathrm{R}+}(k), I^{\mathrm{L}+}(k)$ spectra are already shock-transmission enhanced DS relative to US by a factor of 60 (Figs. 2a,b). Streaming SEPs do not always amplify (damp) waves traveling in the same (opposite) direction, because the $\mu$-distribution is not always monotonic (Figs. $2 \mathrm{e}, \mathrm{f})$. Wave growth and damping do eventually extend to lower $k$.

Do DS inward waves, which scatter SEPs back to the shock with energy loss as seen in outward-wave frame, dominate in the immediate DS and retard acceleration? Figure 1c compares $D_{\mu \mu}^{+}$and $D_{\mu \mu}^{-}$due to outward and inward waves, respectively, of $48.2 \mathrm{MeV}$ protons in the first DS cell at $t=72$ and $576 \mathrm{~s}$. At both times, scattering by strong outward waves dominate in the central $\mu$-range, where most of the SEPs are scattered back and forth across the shock. Hence shock acceleration is not significantly retarded by SEP damping (amplification) of DS outward (inward) waves.

\section{SUMMARY AND DISCUSSION}

Shock acceleration is simulated in a moving box model with the usual processes of SEP transport and momentum diffusion, and the propagation, shock transmission, and SEPdriven evolution of Alfvén waves. For typical coronal conditions and weak ambient waves, a $2500 \mathrm{~km} \mathrm{~s}^{-1}$ parallel shock starting at $3.5 r_{\odot}$ accelerates $18-50 \mathrm{keV}$ DS seed protons at $0.001 n_{\mathrm{H}}$ to a power-law intensity spectrum with $E_{\text {knee }} \sim 300 \mathrm{MeV}$ in $10 \mathrm{~min}$ (Fig. 1a). The box size of $0.215 r_{\odot}=2.8 \times 10^{5}$ ion inertial lengths and $\Omega t=8.2 \times 10^{5}$ for $t=600 \mathrm{~s}$ are respectively 10 and 140 times larger than in the hybrid simulation of Giacalone (2004).

A key feature of the model is the consistent use of the $\mu$-dependent cyclotron resonance condition $B / k \approx P\left(\mu-V_{\mathrm{A}} / v\right)$ to calculate the momentum diffusion tensor $D_{\alpha \beta}$ and the wave growth rate $\gamma_{\sigma}$, so that waves excited by low-energy protons also scatter high-energy ones. Using the $\mu$-dependent condition to calculate $\gamma_{\sigma}$ but the "sharpened" condition $B / k=P$ to calculate $D_{\mu \mu}$ underestimates the effect of amplified waves on $D_{\mu \mu}$, giving little acceleration 
(Berezhko et al. 1998). Using the "sharpened" condition to calculate both $\gamma_{\sigma}$ and $D_{\mu \mu}$ (Vainio \& Laitinen 2007) requires high-energy protons to amplify unique resonant waves from the ambient state before they experience enhanced scattering. The "sharpened" condition is a compromise on the physics of cyclotron resonant interaction.

The evolution of SEPs and DS inward and outward Alfvén waves shows that (a) secondorder Fermi acceleration is not significant, and (b) in the immediate DS, $D_{\mu \mu}^{+}>>D_{\mu \mu}^{-}$ (Fig. 1c) over the $\mu$-range where initial acceleration at $E>E_{k n e e}$ occurs, hence SEP interaction with DS waves do not seriously retard acceleration.

At $t=600 \mathrm{~s}, \mathrm{SEP}$ energy density $\epsilon_{\mathrm{SEP}}=7900 \mathrm{MeV} \mathrm{cm}^{-3}$ at $r_{\mathrm{sh}}=5.65 r_{\odot}$, falling steeply with $x$; shock ramp energy density $\epsilon_{\text {ramp }}=6100 \mathrm{MeV} \mathrm{cm}^{-3}$, Alfvén-wave magnetic energy density $\epsilon_{\text {wave }}=470 \mathrm{MeV} \mathrm{cm}{ }^{-3}$, and $B_{0}^{2} / 8 \pi=510 \mathrm{MeV} \mathrm{cm}^{-3}$. This indicates the shock would be modified, an effect not considered here, and that we are pushing the limits of quasilinear theory. Reducing injection from $b=0.001$ to $b=0.0005$ yields $E_{\text {knee }} \sim 100 \mathrm{MeV}$, $\epsilon_{\mathrm{SEP}}=1950 \mathrm{MeV} \mathrm{cm}^{-3}$ and $\epsilon_{\text {wave }}=420 \mathrm{MeV} \mathrm{cm}^{-3}$. Unfortunately, it is impossible to deduce $j_{E}$ at 1 AU from Figure 1a because of streaming limit (Ng \& Reames 1994; Reames \& Ng 1998). We plan to inject escaping proton flux from this model into an IP transport model with self-consistent waves (e.g., $\mathrm{Ng}$ et al. 2003) to predict $j_{E}$ at $1 \mathrm{AU}$.

Detailed study of model dependence on shock, ambient wave, and plasma parameters will be presented separately in a full paper. Although the model is simplified, neglecting e.g. shock geometry and wave cascading, it captures the essential role of wave-particle interaction with a realistic description of SEP shock acceleration through self-amplified waves. Faster acceleration to higher energy may be possible at quasi-perpendicular shocks. In future, we will include minor ions and the effects of shock geometry, and integrate the box acceleration model into an IP transport model.

This work was supported by NASA under LWS-04-0000-0076 and SHP04-0016-0024.

\section{REFERENCES}

Bell, A. R. 1978, MNRAS, 182, 147

Berezhko, E.G., Petukhov, S.I., \& Taneev, S. N. 1998, Astron. Letters, 24, 122

Bieber, J. W., et al. 1994, ApJ, 420, 294

Cliver, E. W., Kahler, S. W. \& Reames, D. V. 2004, ApJ, 605, 902 
Dulk, G. A. \& McLean, D. J. 1978, Sol. Phys., 57, 279

Giacalone, J. 2004, ApJ, 609, 452

Giacalone, J. 2005, ApJ, 628, L37

Giacalone, J. \& Neugebauer, M. 2008, ApJ, 673, 629

Gordon, B. E., Lee, M. A., Möbius, E., \& Trattner, K. J. 1999, J. Geophys. Res., 104, 28,263

Gosling, J. T. et al. 1981, J. Geophys. Res., 86, 547

Guhathakurta, M., Sittler, E. C. Jr., \& McComas D. 1999, Space Sci. Rev., 87, 199

Lee, M. A. 1982, J. Geophys. Res., 87, 5063

Lee, M. A. 1983, J. Geophys. Res., 88, 6109

Lee, M. A. 2005, ApJS, 158, 38

Li, G., Zank, G. P., \& Rice, W. K. M. 2005, J. Geophys. Res., 110, A06104

Mason, G. M., Mazur, J. E., \& Dwyer, J. R. 1999, ApJ, 525, L133

Ng, C. K., \& Reames, D. V. 1994, ApJ, 424, 1032

Ng, C. K., \& Reames, D. V. 1995, ApJ, 453, 890

Ng, C. K., Reames, D. V., \& Tylka, A. J. 1999, Geophys. Res. Lett., 26, 2145

Ng, C. K., Reames, D. V., \& Tylka, A. J. 2003, ApJ, 591, 461

Ng, C. K. 2007, in AIP Conf. Proc. 932, Turbulence and Nonlinear Processes in Astrohysical Plasmas, ed. D. Shaikh and G. P. Zank (Melville: AIP), 271

Reames, D. V. 1999, Space Sci. Rev., 90, 413

Reames, D. V., \& Ng, C. K. 1998, ApJ, 504, 1002

Reames, D. V., Ng, C. K., \& Tylka, A. J. 2000, ApJ, 531, L83

Tylka, A. J., et al. 2001, ApJ, 558, L59

Tylka, A. J. \& Lee, M. A. 2006, ApJ, 646, 1319

Vainio, R., \& Schlickeiser, R. 1999 A\&A, 343, 303 
Vainio, R. 2001 Proc 27th ICRC, Hamburg, 2054

Vainio, R., \& Laitinen, T. 2007 ApJ, 658, 622

Webb, G. M., Zakharian, A., Brio, M., \& Zank, G. P. 1999, J. Plasma Phys., 61, 295

Wentzel, D. G. 1974, ARA\&A, 12, 71

Zank, G. P., et al. 2001, Phys. Plasmas, 8, 4560

Zank, G. P., et al. 2006, J. Geophys. Res., 111, A06108

Zank, G. P., Li, G. \& Verkhoglyadova, O. 2007, Space Sci. Rev., 130, 225 

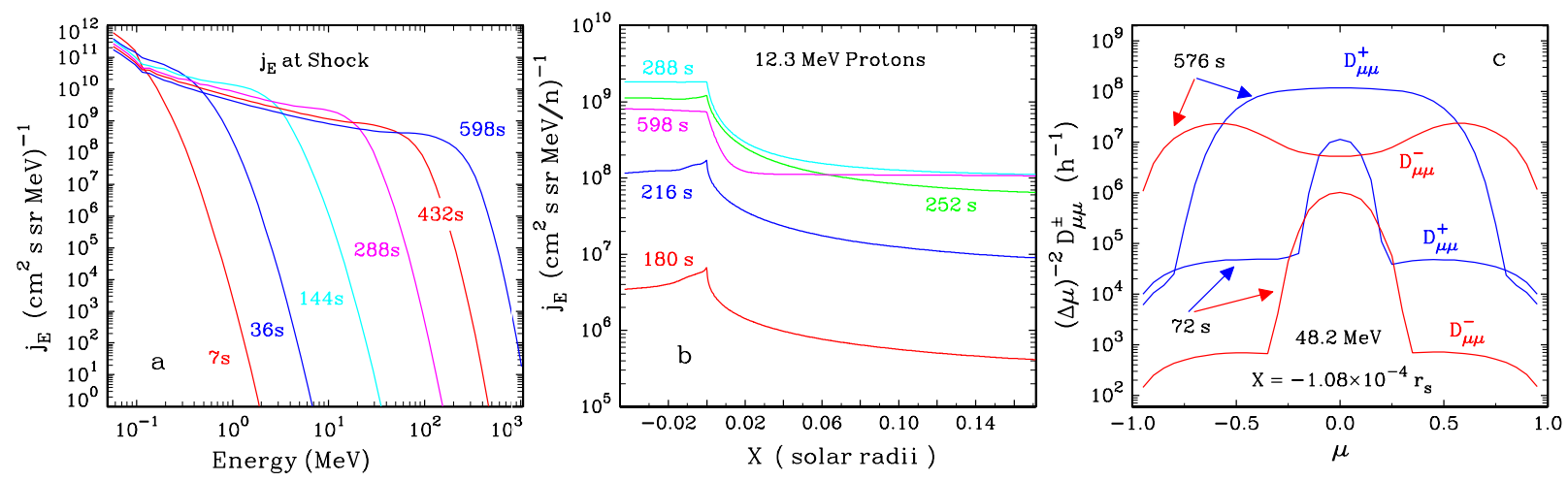

Fig. 1.- (a) Proton intensity spectra at the first cell upstream. (b) $12.3 \mathrm{MeV}$ proton intensity spatial profiles. (c) $48.2 \mathrm{MeV}$ proton $\mu$-diffusion coefficients $D_{\mu \mu}^{ \pm}$vs $\mu$ due to outward and inward Alfvén waves at the first cell downstream at t $=72 \mathrm{~s}$ and 576 s. $\Delta \mu=1 / 20$. 

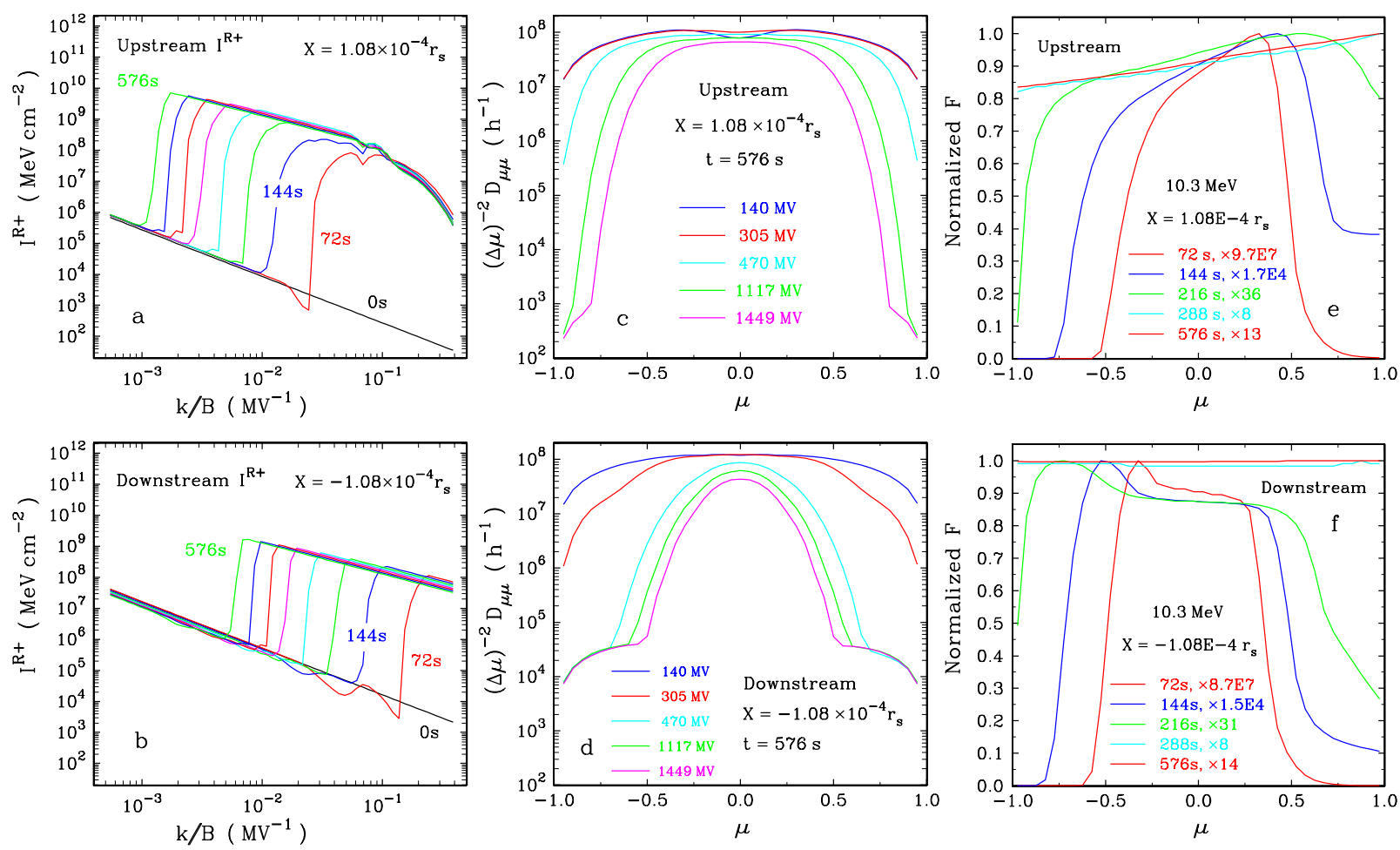

Fig. 2. - Evolution in the first cell upstream of (a) $I^{\mathrm{R}+}$ at $72 \mathrm{~s}$ intervals, (c) $D_{\mu \mu}$ at $\mathrm{t}=576 \mathrm{~s}$ at indicated rigidities, and (e) pitch-angle distributions at indicated times. The lower panels (b), (d), and (f) show the same respectively in the first cell downstream. 